\title{
Editorial
}

\section{Achieving Sustainable 5G}

\author{
Kai Yang $\left(\mathbb{D},{ }^{1}\right.$ Jinsong $W u\left(\mathbb{D},{ }^{2}\right.$ and Nan Yang $\mathbb{D}^{3}$ \\ ${ }^{1}$ The School of Information and Electronics, Beijing Institute of Technology, Beijing 100081, China \\ ${ }^{2}$ The Department of Electrical Engineering, Universidad de Chile, Santiago, Chile \\ ${ }^{3}$ The Research School of Engineering, Australian National University, Canberra, ACT 2601, Australia \\ Correspondence should be addressed to Kai Yang; yangkai@bit.edu.cn
}

Received 27 September 2018; Accepted 27 September 2018; Published 18 October 2018

Copyright (c) 2018 Kai Yang et al. This is an open access article distributed under the Creative Commons Attribution License, which permits unrestricted use, distribution, and reproduction in any medium, provided the original work is properly cited.

Due to the exponentially increased demands of mobile data traffic, e.g., a 1000-fold increase in traffic demand from $4 \mathrm{G}$ to $5 \mathrm{G}$, and the explosive growth in connected mobile devices, dramatic changes in the designs of network architecture are required to meet the $5 \mathrm{G}$ requirements, and the opportunities and challenges of $5 \mathrm{G}$ rapidly attract great attention from academics, industries, and governments. According to the trend in cellular networks evolution, 5G networks will be heterogeneous ones consisting of macrocells along with a large number of small cells, device-to-device pairs, and machine type communication devices based communication tiers [1]. Indeed, to achieve sustainable $5 \mathrm{G}$ and to accelerate the launch of $5 \mathrm{G}$ networks, various promising technologies have been proposed and investigated as essential enablers for the operators to achieve a more efficient use of available radio resource and network infrastructure and to reduce both the capacity expenditure and operation expenditure in the network deployment and operations. The motivation behind this special issue is to solicit cutting-edge research results on achieving sustainable $5 \mathrm{G}$.

The paper "Achievable Rates of Gaussian Interference Channel with Multi-Layer Rate-Splitting and Successive Simple Decoding" proposes a scheme which employs multi-layer rate-splitting (RS) at the transmitters and successive simple decoding (SSD) at the receivers in the two-transmitter and two-receiver Gaussian interference channel (IC) model and then studies the achievable sum capacity of this scheme. Numerical simulations are presented to validate that multilayer RS and SSD are not generally weaker than simultaneous decoding with respect to the achievable sum capacity, at least for some certain channel gain conditions of IC.
The paper "The Rayleigh Fading Channel Prediction via Deep Learning" presents a multi-time channel prediction system based on backpropagation (BP) neural network with multi-hidden layers, which can predict channel information effectively and benefit for massive multiple-input multipleoutput performance, power control, and artificial noiseaided physical-layer security scheme design. Meanwhile, an early stopping strategy to avoid the overfitting of BP neural network is introduced.

The paper "General Multimedia Trust Authentication Framework for 5G Networks" proposes a novel multimedia authentication framework based on trusted content representation (TCR) for $5 \mathrm{G}$ networks. The general framework is suitable for various multimedia contents, e.g., text, audio, and video. The generality of the framework is guaranteed by the TCR technique, which authenticates the contents semantics at both high and low levels.

The paper "A Sparse Temporal Synchronization Algorithm of Laser Communications for Feeder Links in 5G Nonterrestrial Networks" addresses the temporal synchronization problem in laser communications. In this paper, a new sparsity-aware algorithm for temporal synchronization is proposed without carrier aid through sparse discrete polynomial-phase transformation and sparse discrete fractional Fourier transformation.

The paper "Uplink Nonorthogonal Multiple Access Technologies Toward 5G: A Survey" aims to provide a comprehensive overview about the promising nonorthogonal multiple access (NOMA) schemes. The state-of-the-art NOMA schemes are analyzed by comparing the operations applied at the transmitter, and typical multiuser detection algorithms 
corresponding to these NOMA schemes are introduced. In addition, the implementation issues of NOMA are discussed for practical deployment.

The paper "A Novel Query Method for Spatial Data in Mobile Cloud Computing Environment" presents a memorybased spatial data query method that uses the distributed memory file system Alluxio to store data and build a two-level index based on the Alluxio key-value structure. According to the characteristics of Spark computing framework, a data input format for spatial data query is discussed.

The paper "Physical-Layer Channel Authentication for 5G via Machine Learning Algorithm" develops a novel authentication method to detect spoofing attacks without a special test threshold while a trained model is used to determine whether the user is legal or illegal. In addition, a two-dimensional test statistic features authentication model is presented for further improvement of detection rate.

\title{
Conflicts of Interest
}

The guest editors declare that they have no possible conflicts of interest or private agreements with companies.

\section{Acknowledgments}

We would like to thank all the reviewers who have participated in reviewing the articles submitted to this special issue.

\author{
Kai Yang \\ Jinsong $W u$ \\ Nan Yang
}

\section{References}

[1] J. An, K. Yang, J. Wu, N. Ye, S. Guo, and Z. Liao, "Achieving sustainable ultra-dense heterogeneous networks for 5G," IEEE Communications Magazine, vol. 55, no. 12, pp. 84-90, 2017. 


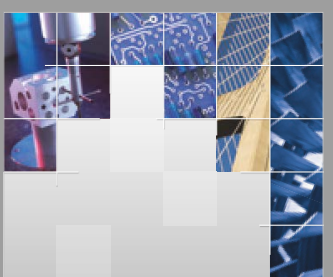

\section{Enfincering}
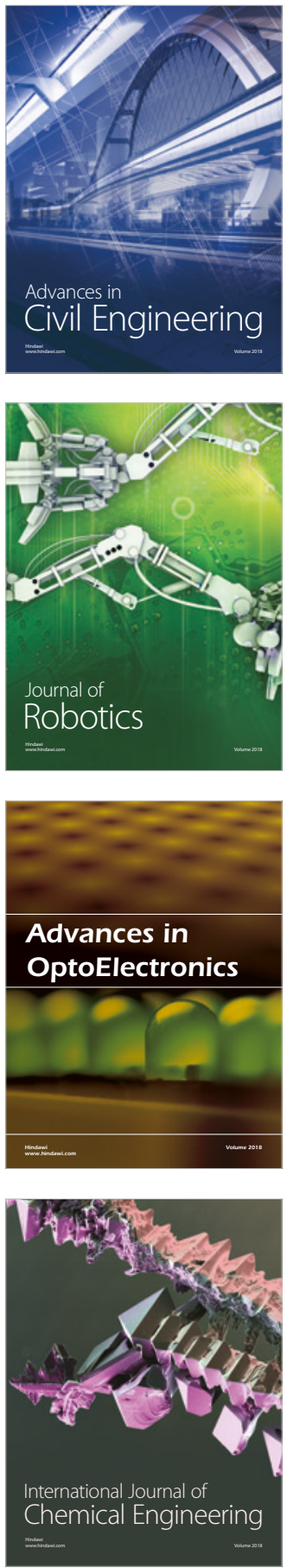

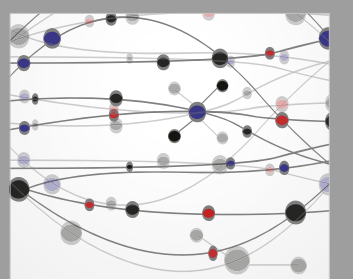

\section{Rotating \\ Machinery}

The Scientific World Journal

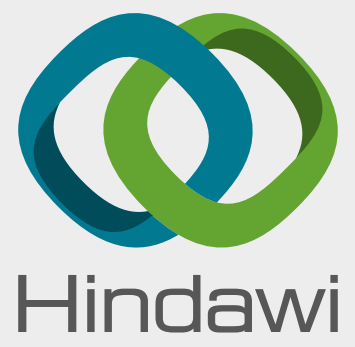

Submit your manuscripts at

www.hindawi.com
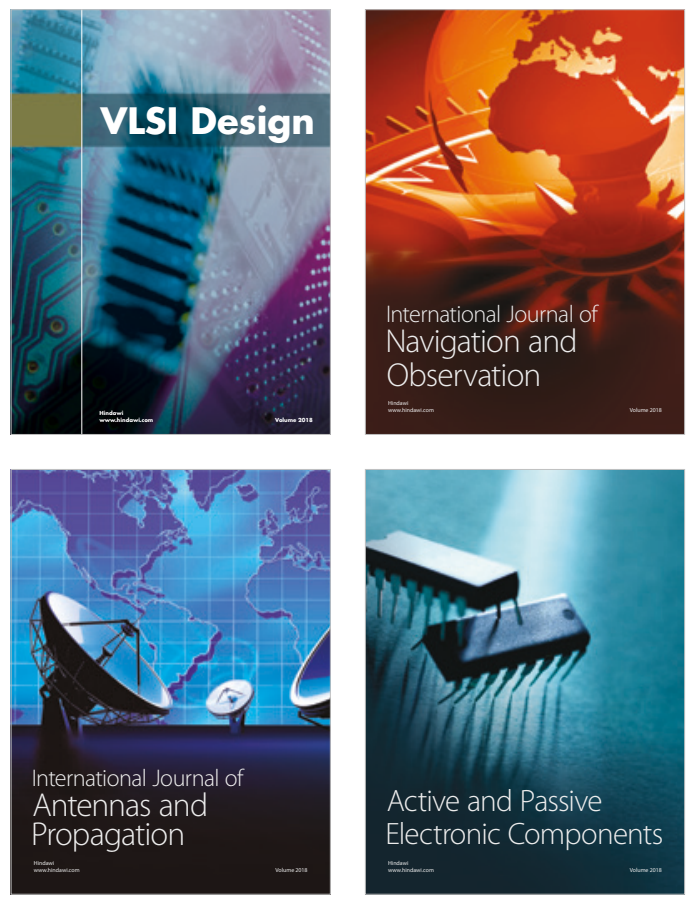
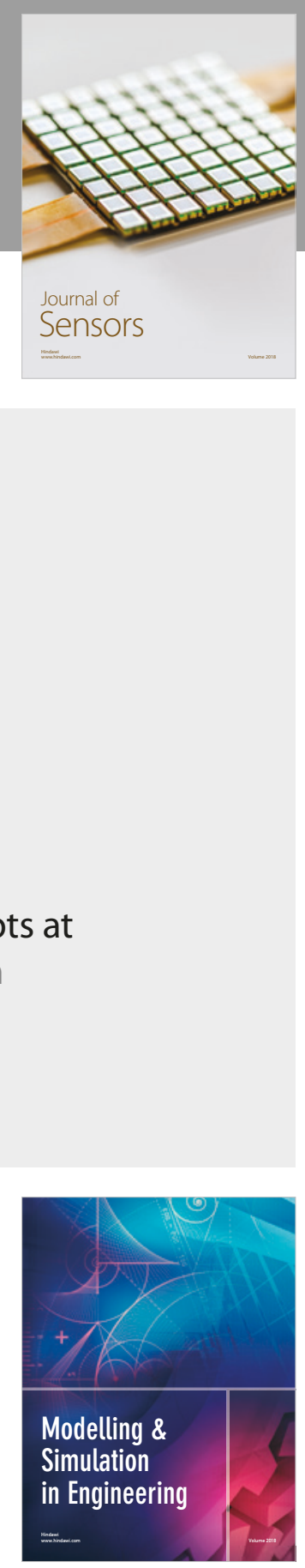

\section{Advances \\ Multimedia}
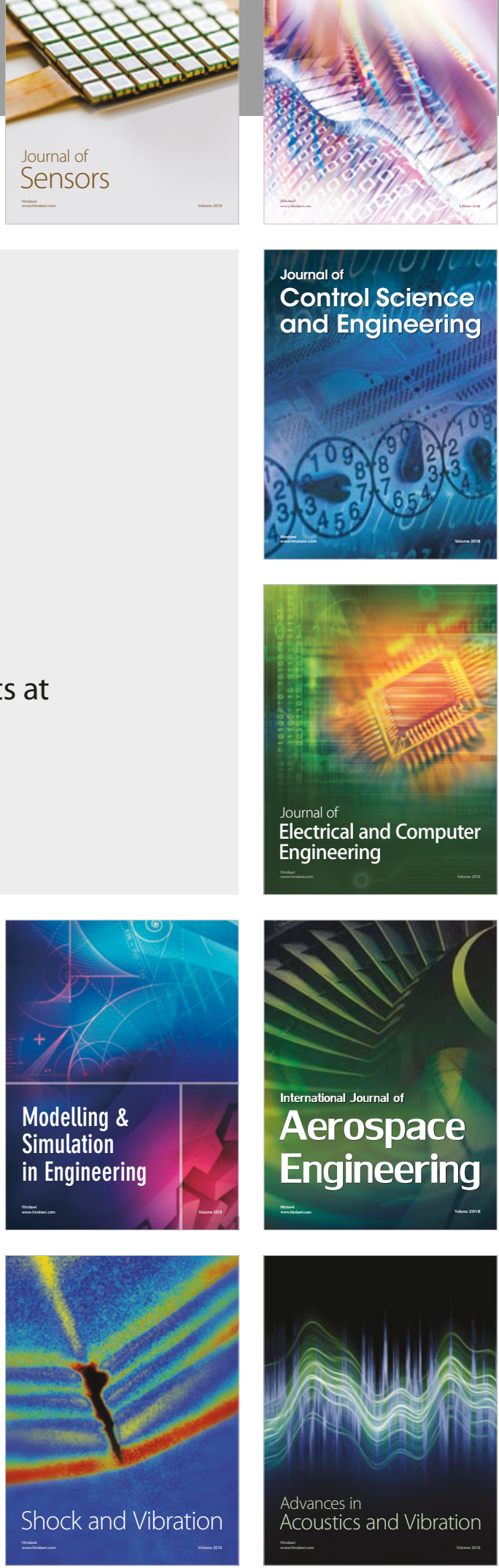